\title{
RAYTEC: a new code for electron cyclotron radiative transport modelling of fusion plasmas
}

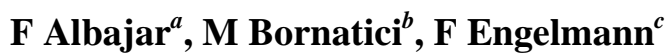 \\ ${ }^{a}$ Fusion for Energy ${ }^{*}$, Josep Pla 2, Barcelona, 08019, Spain \\ ${ }^{b}$ Physics Department “A. Volta”, University of Pavia, Pavia, 27100, Italy \\ ${ }^{c}$ Max-Planck-Institut für Plasmaphysik, Garching, 85748, Germany \\ E-mail: ferran.albajar@f4e.europa.eu
}

\begin{abstract}
As it was recognised that local electron cyclotron (EC) wave power losses can be a competitive contribution to the $1 \mathrm{D}$ electron power balance for reactor-grade tokamak plasmas in regimes as anticipated for steady-state operation, a systematic effort is ongoing to improve the modelling capability for the radial profile of EC wave emission. This effort aims at generating a hierarchy of codes that cover the non-local behaviour of EC wave transport for inhomogeneous plasmas and in the presence of reflecting walls with increasingly improved accuracy and also provide sufficient computational efficiency for being usable in 1D transport studies. The recently developed code RAYTEC which explicitly addresses the geometrical effects present in toroidal plasmas with arbitrary cross-section is described and used to investigate the impact of elongation of the plasma cross section and of toroidicity on the angular dependence of the EC radiation field, on the profile of the net EC wave power density lost from the plasma and on the total EC power loss for ITER-like plasma conditions. Furthermore, a comparison is made with the results of simpler models in use to describe both local and total EC power losses as well as with ones obtained from analytical formulae that are introduced on the basis of Trubnikov's formula for EC power emission.
\end{abstract}

PACS numbers: 52.40.Db, 52.50.-b, 52.55.Dy

\section{Introduction}

Electron cyclotron (EC) wave emission has been shown to be a significant contribution to the local electron power balance of high temperature fusion magnetoplasmas with core electron temperatures of about $35 \mathrm{keV}$ or higher as anticipated for steady-state operation scenarios of ITER and DEMO, see, e.g., Ref. [1]. The estimation of the level of EC wave emission in system and 1D transport studies is usually carried out by using approximate models based on expressions for the global EC emission, see, e.g., Refs. [2,3] or routines like CYTRAN [4] and CYNEQ [5] which, to some extent, incorporate wave transport effects, but assume isotropy of the radiation field.

In order to improve the modelling capability for the profile of the net EC wave power density $d P / d V$ locally emitted (or absorbed), as a first step, recently the accuracy of the Trubnikov [6] and Robinson [7] formulae for the wave absorption coefficients was analyzed [8] and their practical suitability for calculating $d P / d V$ was investigated [9], adopting the code EXACTEC which is based on an exact solution of wave transport for straight rays in a cylindrical plasma with circular cross section and specular wall reflection [10]. As a result, Robinson's formula was found to provide, overall, both good accuracy and numerical efficiency in modelling hot fusion plasmas.

As a second step, a wave transport model analysis has been made for a circularly cylindrical fusion plasma [11] comparing CYTRAN and CYNEQ with EXACTEC and former results from SNECTR [12]. As expected, the assumption of the wave intensity being isotropic made in the former models tends to be a good one if wall reflection is strong and diffuse. Hence, CYTRAN and CYNEQ

\footnotetext{
* The views expressed in this publication are the sole responsibility of the author and do not necessarily reflect the views of Fusion for Energy. Neither Fusion for Energy nor any person acting on behalf of Fusion for Energy is responsible for the use which might be made of the information in this publication.
} 
are fast routines well-suited for transport simulations of fusion plasmas. On the other hand, EXACTEC has confirmed earlier results from SNECTR for specular reflection and in situations in which wall reflection has a diffusive component and, hence, reduces the anisotropy of the radiation field, provides a lower bound (not far from the actual values) to the net EC power density emitted from the hot plasma core as well as to re-absorption in the cool edge plasma.

As a third step, to cover the effects of a non-circular plasma cross-section and of toroidicity, a new code, RAYTEC, tracing the change of the radiation intensity along rays, has been developed. The motivation for this work was to provide an alternative to SNECTR which is no longer in active use. Presently, the code addresses specular reflection of (straight) rays at an ideally toroidal wall which allows tracing rays without having to rely on Monte-Carlo methods (as does SNECTR). The code, primarily intended to be applied to plasmas in thermodynamic equilibrium, can readily be extended to include a fast electron population described by a Maxwellian distribution. The efficiency of the computations has been enhanced by adopting Robinson's formula for the calculation of the EC absorption coefficient as well as by an appropriate modelling of the inner hot plasma for the lower frequencies for which the plasma effectively behaves as a black body and which contributes little to $d P / d V$.

In this paper, we describe the approach adopted in RAYTEC (Sect.2) and report on first results obtained with this code for reactor-grade plasmas (Sect.3). Part of the material has been presented to the Workshop EC-15 as an invited paper [13]. In particular, here we (i) address the question to which extent elongation of the plasma cross section and toroidicity affect the angular distribution of the radiation field (Sect.3.1), (ii) determine, in Sect.3.2, the radial profile of the net EC wave power density $d P / d V$ that is emitted from the plasma for ITER-like plasma parameters and profiles, study the impact of elongation and toroidicity on $d P / d V$ and compare with profiles generated by the frequently used simpler models for $d P / d V$, viz., CYTRAN [4] and EXACTEC [10], as well as with an explicit analytical formula for $d P / d V$ in terms of local and global plasma parameters based on Trubnikov's expression for the total EC power loss [2,6], referred to as LATF, which is described in detail in the Appendix, and (iii) consider the total EC power loss and its dependence on both the plasma geometry and the shape of the plasma temperature as following from this local analysis and also compare the results with those obtained from the total power loss formulae given by Fidone et al [14] and by Albajar et al. [3] (Sect.3.3).

\section{The RAYTEC approach}

For plasmas in thermodynamic equilibrium, the net EC wave power per unit volume radiated at the position $\mathbf{r}$ is

$$
\frac{d P(\mathbf{r})}{d V}=\sum_{\sigma=\mathrm{X}, \mathrm{O}} \int_{\omega_{\min }^{\sigma}}^{\infty} \mathrm{d} \omega \int d^{2} \Omega_{\hat{s}} \alpha^{\sigma}(\mathbf{r}, \omega, \hat{\mathbf{s}})\left[I_{\mathrm{bb}}^{\sigma}(\mathbf{r}, \omega)-I^{\sigma}(\mathbf{r}, \omega, \hat{\mathbf{s}})\right]
$$

with $\omega$ the wave frequency, $\sigma=\mathrm{X}, \mathrm{O}$ the extraordinary and ordinary polarization modes, $d^{2} \Omega_{\hat{s}}$ the element of solid angle around the ray direction $\hat{\mathbf{s}}, \alpha$ is the absorption coefficient, and $I_{b b}^{\sigma}(\mathbf{r}, \omega) \equiv\left(N_{r}^{\sigma}\right)^{2} \omega^{2} T(\mathbf{r}) / 8 \pi^{3} c^{2}$ the black-body intensity for the (local) electron temperature $T(\mathbf{r})$ and a (local) ray refractive index $N_{r}^{\sigma}$. In Eq.(1), the specific radiation intensity $I^{\sigma}$ is governed by the (steady-state) radiative transfer equation (RTE), see, e.g., $[2,10]$, which in the context of geometrical optics accounts for the non-local nature of the radiation process that is due to both re-absorption of radiation and reflection at the wall surrounding the plasma. An analytical solution for $I^{\sigma}$ was obtained for a system having (circularly) cylindrical symmetry and assuming that the reflection at the wall is specular [10]. In toroidal geometry, the poloidal symmetry of the radiation intensity is lost because of (i) the inhomogeneity of the toroidal magnetic field along the horizontal coordinate which enhances emission and absorption at the high-field side of the plasma, (ii) the impact on the ray path length, which affects the effective optical thickness of the plasma, and (iii) the variation of the angle of propagation relative to the magnetic axis along the ray path. Elongation of the plasma cross-section also modifies the ray path lengths and, hence, the effective optical thickness of the plasma. 
For arbitrary geometry and plasma profile, integration of the RTE from a point $\mathbf{r}_{w, 1}$ at the wall to an arbitrary point $\mathbf{r}$ within the plasma along a ray yields

$$
\frac{I(s(\mathbf{r}))}{N_{\mathrm{r}}^{2}}=I_{\mathrm{ref}}\left(s\left(\mathbf{r}_{\mathrm{w}, 1}\right)\right) e^{-\tau\left(s(\mathbf{r}), s\left(\mathbf{r}_{\mathrm{w}, 1}\right)\right)}+i\left(s(\mathbf{r}), s\left(\mathbf{r}_{\mathrm{w}, 1}\right)\right)
$$

where

$$
i\left(s(\mathbf{r}), s\left(\mathbf{r}_{\mathrm{w}, 1}\right)\right)=\frac{\omega^{2}}{8 \pi^{3} c^{2}} \int_{s\left(\mathbf{r}_{\mathrm{w}, 1}\right)}^{s(\mathbf{r})} d s^{\prime} T\left(s^{\prime}\right) \alpha\left(s^{\prime}\right) e^{-\tau\left(s(\mathbf{r}), s^{\prime}\right)},
$$

$\tau$ is the optical distance along the ray path, and $s$ is a coordinate along the ray trajectory (the dependencies on $\sigma, \omega$, and $\hat{\mathbf{s}}$ have been dropped for convenience). In Eq.(2), $I_{\text {ref }}$ refers to the radiation intensity after reflection. For specular wall reflection, the incident and reflected intensities at the point $\mathbf{r}_{w, 1}$ are related by

$$
I_{\text {ref }}\left(s\left(\mathbf{r}_{\mathrm{w}, 1}\right)\right)=R_{\mathrm{w}} I_{\text {inc }}\left(s\left(\mathbf{r}_{\mathrm{w}, 1}\right)\right)
$$

(disregarding polarization scrambling, for simplicity) where $R_{\mathrm{w}}$ is the (effective) wall reflection coefficient $\left(0 \leq R_{\mathrm{w}} \leq 1\right)$. The two terms of the right-hand side of Eq.(2) describe the re-absorption and the (effective) emission of radiation over the ray path between $\mathbf{r}_{\mathrm{w}}$ and the investigated position $\mathbf{r}$.

By applying Eqs.(2) and (4) in a recurrent way between two consecutive reflection points $\mathbf{r}_{\mathrm{w}, \mathrm{k}}$ and $\mathbf{r}_{\mathrm{w}, \mathrm{k}+1}$ on the ray path, taken to be piecewise straight with specular reflection at an ideally toroidal wall as illustrated in Fig.1, in backward direction over $n(n \geq 2)$ reflections, yields

$$
\begin{aligned}
I_{\text {ref }}(1)= & R_{\mathrm{w}}^{\mathrm{n}-1} I_{\text {ref }}(n) \exp \left(-\sum_{k=1}^{n-1} \tau(k, k+1)\right) \\
& +\sum_{k=2, n-1} R_{\mathrm{w}}^{\mathrm{k}} i(k, k+1) \exp \left(-\sum_{j=1}^{k-1} \tau(j, j+1)\right)+R_{\mathrm{w}} i(1,2)
\end{aligned}
$$

with $i(k, k+1)$ given by applying Eq.(3) to $\mathbf{r}_{\mathrm{w}, \mathrm{k}}$ and $\mathbf{r}_{\mathrm{w}, \mathrm{k}+1}$. Note that the ray direction $\hat{\mathbf{s}}$ is changed in every reflection depending on the geometry of the first wall (or, more generally, on the local properties of the wall at the reflecting point). For a sufficiently high number $n$ of reflections, the optical length of the ray becomes much larger than one, $\sum_{k=1}^{n-1} \tau(k, k+1) \gg 1$, and the specific intensity does no longer depend on its initial value which then can be taken $I_{\text {ref }}(n)=0$, for simplicity. The number of reflections to be considered depends on the plasma dimension and parameters (temperature, density and magnetic field), but also on the wave frequency and ray direction. Specifically in an optically thick situation, a small number of reflections already provide a good estimate of $I_{\text {ref }}(1)$.

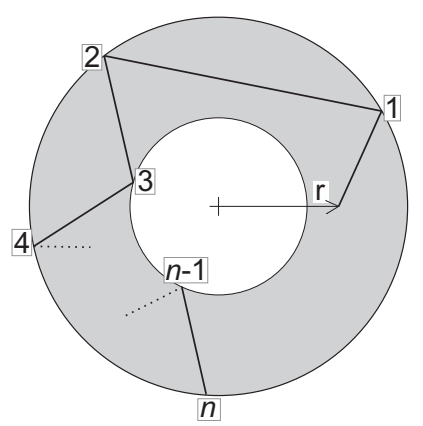

Figure 1. Schematic view of a zigzag ray in the equatorial plane of a plasma torus for specular wall reflection.

The described method to calculate the specific intensity for an arbitrary plasma and first-wall geometry has been implemented in the RAYTEC code. In contrast to the approach followed in 
SNECTR [12] in which the net power density radiated is calculated from the difference between the radiation intensity emerging from and entering through the surface of a given plasma region when launching $\mathrm{n}$ random rays from the plasma wall to the inner plasma, in RAYTEC $d P / d V$ is evaluated at every plasma position $\mathbf{r}$ using the exact expression (1). For the applications reported here, a simplified plasma structure has been adopted in which the magnetic surfaces are taken to be concentric ellipses.

The efficiency of the computation in the RAYTEC code has been enhanced by adopting Robinson's formula for the calculation of the EC absorption coefficient $[7,8,9]$ and by an appropriate modelling in the inner hot plasma for the low frequencies for which the plasma is a black body. In fact, the computer time needed to calculate the local net EC power loss in the low-frequency part of the wave spectrum, contributing little to $d P / d V$, is significantly longer than for higher frequencies. This is due to the slow convergence of the integration in Eq.(1) for low frequencies where emission exceeds absorption only marginally and, in addition, the spectrum has a line-like structure. For these low frequencies, in RAYTEC the diffusion limit of radiative transfer, see, e.g., [10], is used for which one has

$$
{\frac{d^{2} P}{d V d \omega}}^{\sigma}(\mathbf{r}, \omega) \simeq-\left(N_{r}^{\sigma}\right)^{2} \frac{\omega^{2}}{8 \pi^{3} c^{2}}\left(\int d^{2} \Omega_{\hat{s}} \frac{\hat{\mathbf{s}} \hat{\mathbf{s}}}{\alpha^{\sigma}(s(\mathbf{r}), \omega, \hat{\mathbf{s}})}\right): \nabla \nabla T(\mathbf{r}) .
$$

This approximation is valid for the frequency range

$$
\omega_{\min }^{\sigma}(\hat{\mathbf{s}}) \leq \omega \leq \omega_{b b}^{\sigma}(\hat{\mathbf{s}})
$$

where $\omega_{\min }^{\sigma}$ is the lower limit of the $\omega$-integration in Eq.(1) and $\omega_{b b}^{\sigma}$ is the upper bound of the frequency range for which the plasma effectively radiates as a black-body determined by $\omega_{b b}^{\sigma}(\hat{\mathbf{s}})=\min \left\{\omega_{1}^{\sigma}(\hat{\mathbf{s}}), \omega_{2}^{\sigma}(\hat{\mathbf{s}})\right\}$ with, respectively, $\omega_{1}^{\sigma}(\hat{\mathbf{s}})$ and $\omega_{2}^{\sigma}(\hat{\mathbf{s}})$ the upper limits of the frequency ranges for which, at the position $\mathbf{r}$, the optical distance from the wall is large, $\tau^{\sigma}(\omega, \hat{\mathbf{s}}) \gg 1$, and that for which the radiative mean free path $(1 / \alpha)$ is short compared to the scale-length of the temperature variation, $\left|\frac{1}{\alpha^{\sigma}(s(\mathbf{r}), \omega, \hat{\mathbf{s}})} \frac{1}{\mathrm{~T}} \frac{d T}{d s}\right|<1$. Note that the frequency range (7) depends on the mode $\sigma$, the position $\mathbf{r}$ (not explicitly indicated for convenience), and the ray direction $\hat{\mathbf{s}}$. The frequencies $\omega_{1}^{\sigma}(\hat{\mathbf{s}})$ and $\omega_{2}^{\sigma}(\hat{\mathbf{s}})$ limiting the validity of the diffusive approximation, Eq.(6), from above, quantified, respectively, by the definitions $\tau^{\sigma}(\omega, \hat{\mathbf{s}})=3$ (referred to as $\left.\left.\omega_{1}^{\sigma}(\hat{\mathbf{s}})\right|_{3}\right)$ and $\left|\frac{1}{\mathrm{~T}} \frac{d T}{d s}\right|=0.3 \alpha^{\sigma}(s, \omega, \hat{\mathbf{s}})$ (referred to as $\left.\omega_{2}^{\sigma}(\hat{\mathbf{s}})\right|_{0.3}$ ), are shown in Figs.2 for the position $\rho=0.05$ in the plasma core as well as for $\rho=0.5$ intermediate between core and edge on the outboard side, as a function of the angle $\theta$ between the ray direction and the magnetic field for both the extraordinary $(\sigma=X)$ and the ordinary $(\sigma=\mathrm{O})$ mode. It appears that in the core plasma the most stringent condition for the application of the diffusion approximation is $\tau^{\sigma}(\omega, \hat{\mathbf{s}}) \gg 1$. Towards the edge both conditions become more restrictive: already for $\rho=0.5$ the approximation only applies to the lowest harmonic frequencies; adjacent to and in the edge region itself the diffusion model does not apply to any frequency. In the hot plasma core where most of the radiation is emitted, typical values of the frequency limit $\omega_{b b}^{\sigma}(s)$ for the hightemperature ITER case with an advanced $T$ profile, as analysed in the following section, are 3.5-4.5 times the EC frequency $\omega_{\mathrm{c}}$ for the extraordinary mode $(\sigma=\mathrm{X})$ and $(2-3) \omega_{\mathrm{c}}$ for the ordinary mode $(\sigma=\mathrm{O})$; it is seen from the left part of Fig.3a where the frequency spectrum of the specific intensity $I^{\sigma}$ is shown that in these frequency ranges the $I^{\sigma}$ practically coincide with the local black-body intensity. From the right part of Fig.3a where the frequency dependence of the net EC radiative power density $d^{2} P / d V d \omega$ at $\rho=0.05$ is plotted, it is transparent that the contribution from the frequency range (7), 
that is $\omega_{b b}^{\mathrm{O}}(\hat{\mathbf{s}}) \lesssim 2.5 \omega_{c}$ and $\omega_{b b}^{\mathrm{X}}(\hat{\mathbf{s}}) \lesssim 4.2 \omega_{c}$, to $d^{2} P / d V d \omega$ there is, in fact, negligibly small. This latter conclusion equally applies to the cooler plasma further outside (see Figs.3b for $\rho=0.5$ on the outboard side). Figs. $3 \mathrm{~b}$ also show that for rays propagating towards the low-field side, that is from hotter to cooler regions, the specific intensity $I^{\sigma}$ is larger than the local black-body intensity for the lower frequencies where the plasma as a whole tends to be effectively optically thick. Hence for such conditions power reabsorption prevails, implying a weakened overall power emission at these lower frequencies. This tendency gradually strengthens with increasing $\rho$ so that, adjacent to the edge, absorption of the $\mathrm{EC}$ wave power can become the dominant process yielding a reversal of the profile of the net EC power density $d P / d V$ as observed earlier $[1,10,12]$.
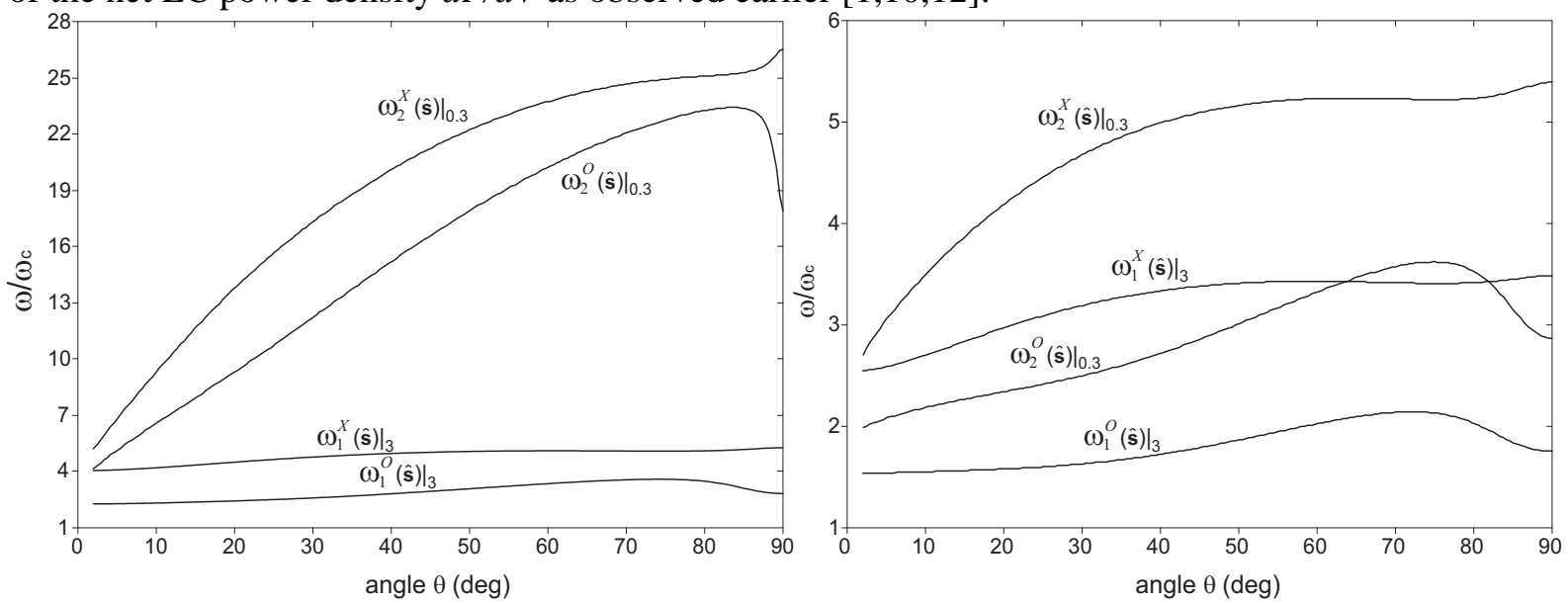

Figure 2. Typical limiting frequencies $\omega_{1}^{\sigma}(\hat{\mathbf{s}})$ and $\omega_{2}^{\sigma}(\hat{\mathbf{s}})$ for rays in the equatorial plane pointing towards the low-field side as a function of the angle $\theta$ between the ray direction and the magnetic field (ITER-like plasma parameters, the reference plasma geometry and the advanced $T$ profile (see Sect.3) ) in the plasma core $(\rho=0.05)$, left plot, and at $\rho=0.5$ on the outboard side, right plot.

The low-frequency waves travelling in a toroidal plasma towards the high-field side are cut in RAYTEC when approaching the cut-off frequency. This treatment has been shown to be robust compared to other approximations. The contribution of these waves to $d P / d V$ is likewise negligible.

The RAYTEC code has been validated against both the exact code EXACTEC in the limit of circularly cylindrical plasmas and a preliminary version of the code ("proto-RAYTEC"), written for cylindrical geometry and elliptical cross section, producing identical results.
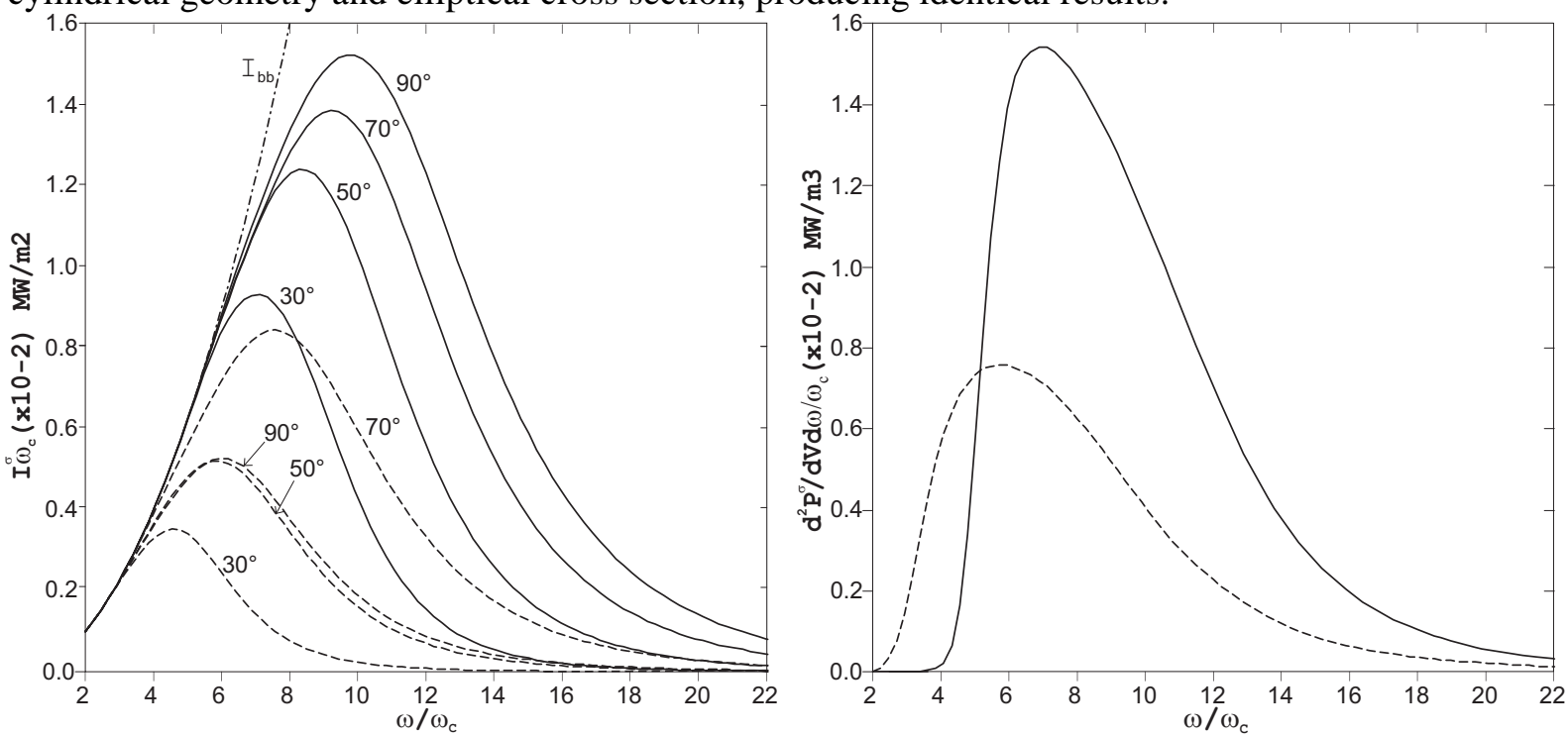

Figure 3a. Frequency spectrum of the specific intensity $I^{\sigma}$ (left plot) for rays in the equatorial plane propagating towards the high-field side at various angles $\theta\left(\theta=30^{\circ}, 50^{\circ}, 70^{\circ}\right.$ and $\left.90^{\circ}\right)$ relative to the 
magnetic field, and of the net EC radiative power density $d^{2} P / d V d \omega / \omega_{c}$ (right plot) in the plasma core $(\rho=\mathrm{r} / a=0.05)$ : X-mode (solid curves), O-mode (dashed curves); in the left plot the black-body intensity is also shown (dot-dashed curve); ITER-like plasma parameters, the reference plasma geometry, the advanced $T$ profile (see Sect.3) and a wall reflectivity $R_{\mathrm{W}}=0.8$ are taken.
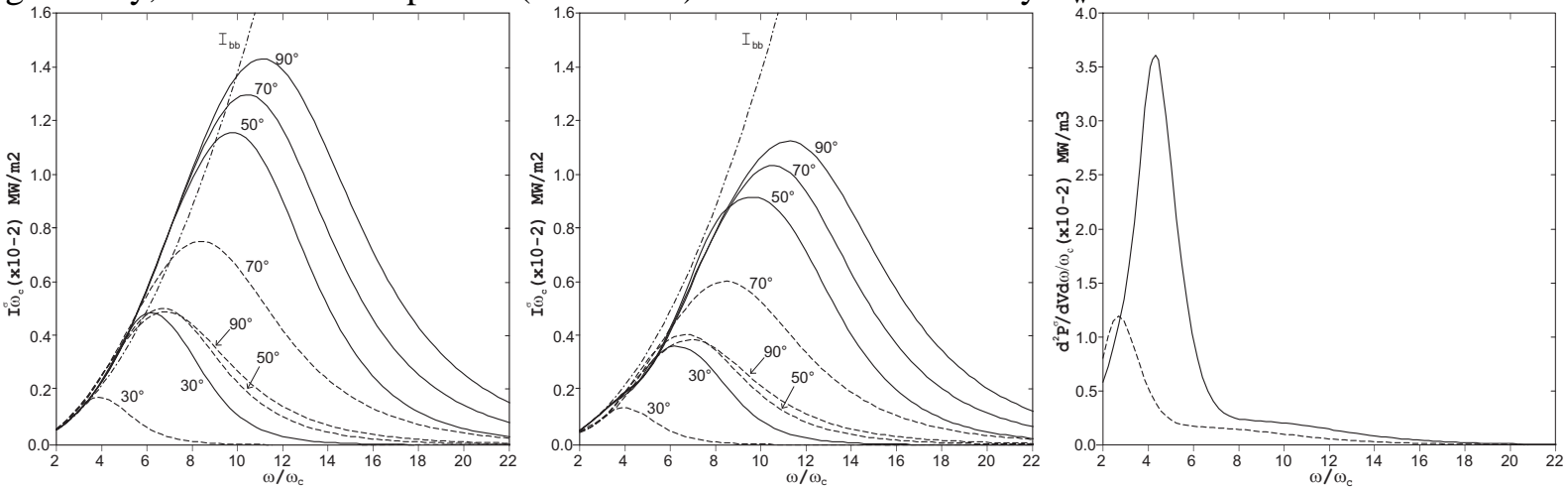

Figure 3b. Same as Fig.3a, but for $\rho=0.5$ on the outboard side; the left and the middle plot refer, respectively, to rays propagating to the low-field and high-field side.

\section{RAYTEC studies}

RAYTEC has been used to investigate the impact of the plasma geometry (vertical elongation and toroidicity) on the EC radiation that is emitted from a reactor-grade plasma. Specific issues addressed are to which extent geometry effects by themselves (i) are able to reduce the anisotropy of the emitted radiation, (ii) affect the profile of the net emitted EC wave power density $d P / d V$, and (iii) influence the total EC power $P_{E C}$ lost from the plasma. In the context of the investigations on the latter two questions, also the appropriateness of simpler models, viz., CYTRAN [4], EXACTEC [10] and a slightly modified Trubnikov formula [2,6] applied locally (to be referred to as LATF; see Appendix for details), was tested; as for the total EC power $P_{E C}$, also the formula given by Albajar et al [3] and that proposed by Fidone et al [14] were considered.

For these studies, ITER-like parameters have been adopted. In the reference case, the plasma geometry was given by a plasma minor radius $a_{r e f}=2 \mathrm{~m}$, a major radius $R_{r e f}=6.2 \mathrm{~m}$, that is an aspect ratio $A_{\text {ref }}=R_{\text {ref }} a_{\text {ref }}=3.1$, and a vertical elongation $\kappa_{\text {ref }}=b_{r e f} a_{\text {ref }}=1.7$. The toroidal magnetic field on the magnetic axis was taken to be $\mathrm{B}=5.3 \mathrm{~T}$. For simplicity, the magnetic surfaces, characterized by the normalized radial coordinate $\rho=r / a$, were assumed to be concentric ellipses and the poloidal magnetic field was disregarded. The profiles of the electron density $n$ and temperature $T$ have been taken in the form $n(\rho)=n(0)\left(1-\rho^{2}\right)^{\gamma_{n}}$ with a peak density $n(0)=1.1 \times 10^{20} \mathrm{~m}^{-3}$ and $\gamma_{\mathrm{n}}=0.1$, corresponding to a volume average density of about $10^{20} \mathrm{~m}^{-3}$, and $T(\rho)=(T(0)-T(1))\left(1-\rho^{\beta_{T}}\right)^{\gamma_{T}}+T(1)$. Two temperature profiles with a peak and an edge temperature $T(0)=45 \mathrm{keV}$ and $T(1)=0.01 \mathrm{keV}$, respectively, and a volume average temperature of 18 $\mathrm{keV}$ were considered: a profile flat in the core plasma with a steep gradient around $\rho=0.6$ which simulates steady-state operation conditions $\left(\gamma_{\mathrm{T}}=8.0, \beta_{\mathrm{T}}=5.4\right.$; in the following referred to as 'advanced' profile) and, for comparison, a 'parabola-like' one (characterized by $\gamma_{\mathrm{T}}=1.5, \beta_{\mathrm{T}}=2.0$ ). Note that increasing the edge temperature $T(1)$ to the $\mathrm{keV}$ range has little influence on the results (see Ref. [11]). For the wall reflection coefficient mostly the modestly high (though representative, see Ref. [1]) value $R_{\mathrm{w}}=0.8$ was taken, but cases without wall reflection $\left(R_{w}=0\right)$ were also considered.

Changes in the plasma geometry as a function of elongation $\kappa$ and aspect ratio $A$ were done keeping the plasma cross section

$$
\pi \kappa a^{2}=\pi \kappa_{\text {ref }} a_{\text {ref }}^{2}
$$


or, equivalently, the plasma volume $V$ divided by $R$, constant. This ensures that effects due to a changing plasma volume in the net wave power density $d P / d V$ and in the total EC power emitted per toroidal length $P_{E C} / R$ are avoided. As a consequence, the major radius $R$ has to be scaled like

$$
R \equiv A a=A a_{\text {ref }} \sqrt{\kappa_{\text {ref }} / \kappa}
$$

when $\kappa$ or $A$ is varied.

\subsection{Angular dependence of EC radiation within the plasma}

Recently, it has been shown that for specular wall reflection in circularly cylindrical geometry the anisotropy of the EC radiation field is considerable for all wall reflection coefficients of practical significance [11] (see also Figs.3, left and middle plots). Therefore it is of interest to investigate to which extent plasma elongation and toroidicity, which both break the symmetry in the poloidal angle $\varphi$, by itself cause an isotropisation of the radiation.

Using RAYTEC, for the ITER-like plasma with an advanced profile as defined above and a wall reflection coefficient $R_{w}=0.8$, the angular dependence of the EC radiation field was investigated at the magnetic axis $(\rho=0)$ and at $\rho=0.5$ on the outboard side, for elongations in the range $\kappa=1$ to 2.05 , the reference value of the aspect ratio $A_{\text {ref }}=3.1$ as well as a large aspect ratio $(A=1000)$ corresponding effectively to the cylindrical case, and two relevant frequencies $\left(\omega / \omega_{c}=5\right.$ and 10$)$. As the plasma emissivity is dependent on the angle $\theta$ between the ray direction and the magnetic field, particular attention was given to the $\theta$-dependence of the specific intensity $I^{\sigma}$. Both rays in the equatorial plane and ones forming an angle of $45^{\circ}$ with that plane were considered. In addition, the dependence of $I^{\sigma}$ on the poloidal angle at fixed $\theta$ was looked at.

Overall, the conclusion is that elongation and toroidicity by themselves do not lead to a sizeable isotropisation of the radiation intensity. For this, a significant diffusive component in the reflection at the wall is necessary. For specular wall reflection the effects of elongation generally are found to be weak: there is a slight tendency for the specific intensity to decrease with increasing elongation, corresponding to a decrease of the effective optical thickness of the system when the horizontal minor radius $a$ decreases according to the scaling of Eq.(8a). Toroidicity, due to the variation of the angle between the ray direction and the magnetic field, tends to mitigate the $\theta$-dependence of $I^{\sigma}$, in particular for small $\theta$ (if $I^{\sigma}$ is close to the black-body value for most $\theta, I^{\sigma}$ is even raised strongly to approach that value), but introduces abrupt changes of $I^{\sigma}$ with $\theta$ when the path length towards the last wall reflection point (and possibly previous ones) discontinuously changes and the optical distance to that reflection point is not large compared to 1. From Figs.3, the considerable anisotropy of $I^{\sigma}$ in the core plasma for propagation in the equatorial plane towards the high-field side is transparent. Further typical examples are shown in Figs.4a and $4 \mathrm{~b}$ which specifically refer to rays in the equatorial plane propagating towards the low-field side. From Fig.4a one also can notice again that in the cooler outside plasma (see the case $\rho=0.5$ outboard) the specific intensity of the radiation does exceed the local black-body value for frequencies for which the plasma as a whole is effectively optically thick while this is not the case for higher frequencies (see Fig.4b). The general tendency of $I^{\sigma}$ to increase in toroidal geometry with respect to the cylindrical one, observed in the plasma core for propagation towards the low-field side, has to do with the inhomogeneity of the magnetic field which enhances emission along the ray path. Moreover, one sees that, further outboard, toroidicity via a reduction of the effective optical thickness by the inhomogeneity of the magnetic field, can lead to a decrease of $I^{\sigma}$ compared to cylindrical geometry, this inverse effect being particularly strong for small angles $\theta$. Finally, the appearance of abrupt changes of $I^{\sigma}$ as caused by path lengths changes between reflection points in toroidal geometry when $I^{\sigma} I^{\sigma}{ }_{b b}$ is smaller than 1 , is evidenced. 

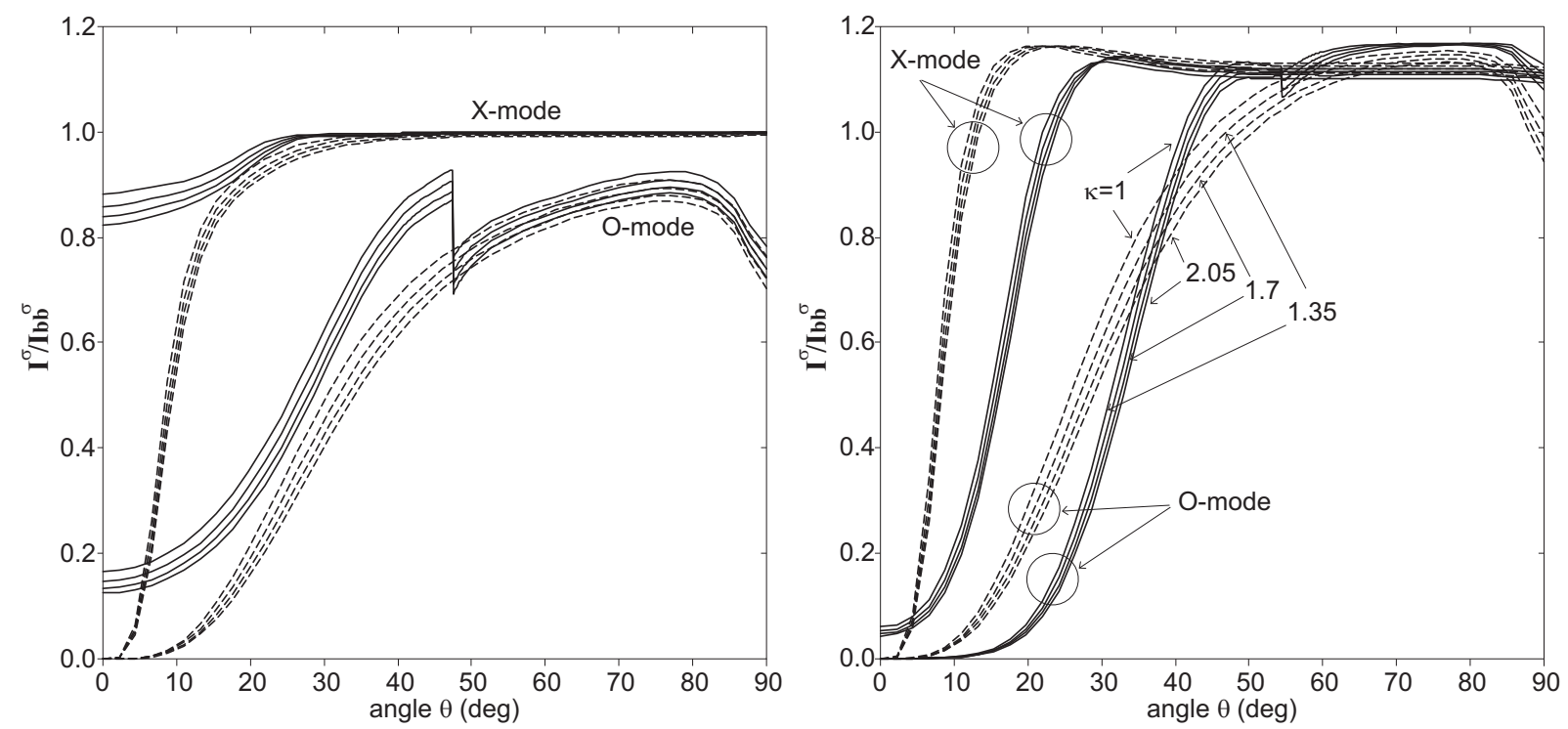

Figure 4a. Specific intensity $I^{\sigma}$ normalized to the black-body value $I_{b b}^{\sigma}$ for the X-and O-mode, $\omega / \omega_{\mathrm{c}}=5$ and rays in the equatorial plane propagating towards the low-field side, for radial positions $\rho=0$ (left plot) and $\rho=0.5$ on the outboard side (right plot), as a function of the angle $\theta$ between the ray direction and the magnetic field; ITER-like parameters, the advanced plasma profile, elongations between $\kappa=1$ and 2.05 (the sequence indicated in the right plot for the O-mode applies to all cases), the reference aspect ratio $A_{r e f}=3.1$ (solid curves) as well as the cylindrical limit $(A=1000$; dashed curves), and a reflection coefficient $R_{w}=0.8$ are considered.
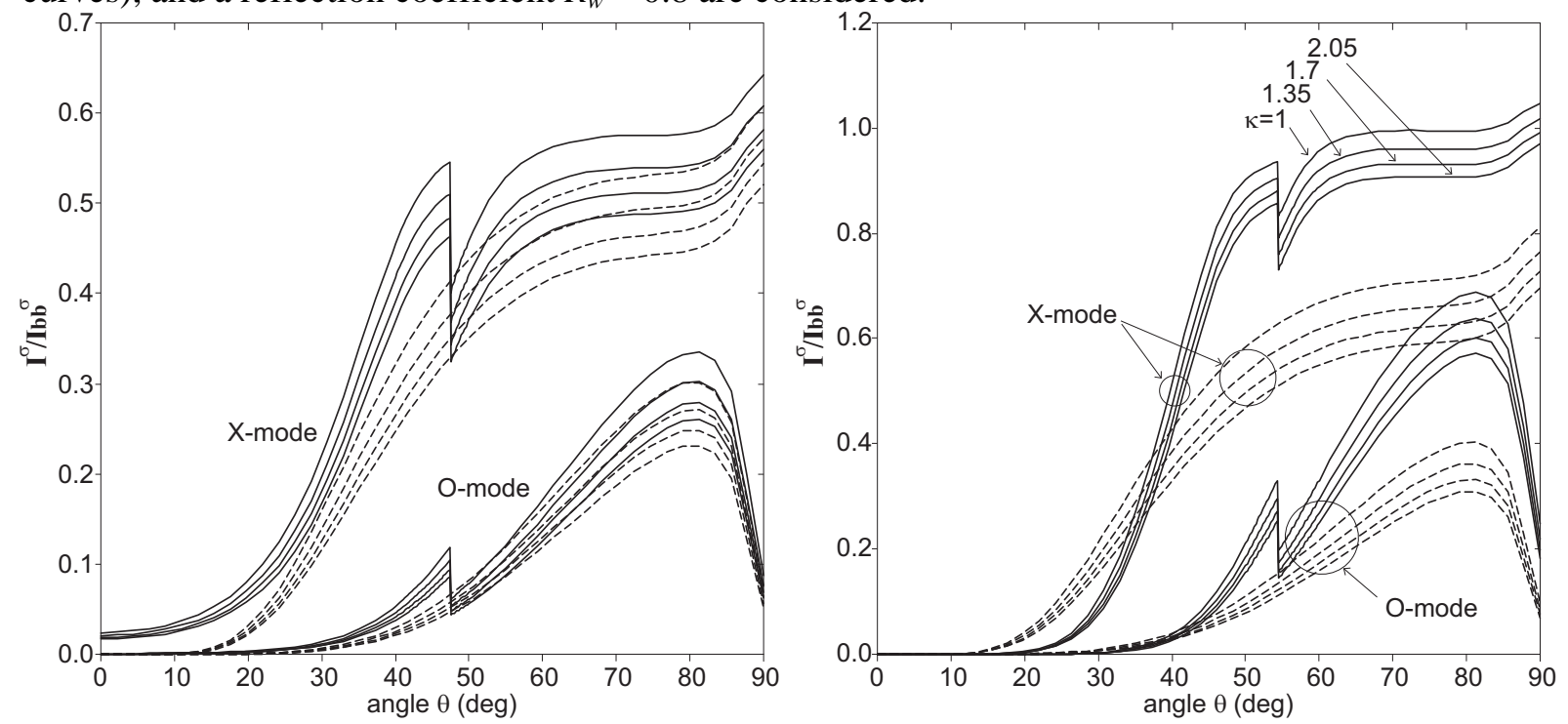

Figure 4b. Same as figure $4 a$, but for $\omega / \omega_{c}=10$.

\subsection{Radial profile of net EC power emission}

The radial profiles of the net EC power density $d P / d V$ emitted, see Eq.(1), as obtained from the RAYTEC code are shown in Fig.5 for the reference geometry, the 'advanced' $T$-profile, and positions at different poloidal angles $\varphi$ on the magnetic surface defined by the radial position $\rho$. The angle $\varphi$ is counted counterclockwise from the horizontal pointing towards the low-field side. As expected, at a given $\rho$, in the inner plasma $d P / d V$ increases from $\varphi=0$ to $\varphi=\pi$, that is towards higher fields the EC emission is enhanced, the enhancement being maximum for intermediate plasma radii, $0.3 \leq \rho \leq 0.4$ :

$d P / d V$ for $\varphi=\pi$ here is about twice the one for $\varphi=0$. From Fig.5 it is also seen that the profile reversal occurring in the edge plasma due to the accumulation of radiation caused by wall reflection, is significantly enhanced on the high-field side. The degree of increase of $d P / d V$ with poloidal angle is slightly reduced around the vertical direction, $\varphi=\pi / 2$, due to an increase of the effective plasma 
dimension connected with elongation. Fig.5 shows that the $d P / d V$ profile averaged over poloidal angles, closely follows the one for $\varphi=\pi / 2$, the extrema of the former being somewhat more pronounced. Similar results have been obtained for other plasma parameters and temperature profiles. Note that there is a sizeable contribution of the O-mode to the EC radiation radiation field and to $d P / d V$ (see Figs.3 and 4).
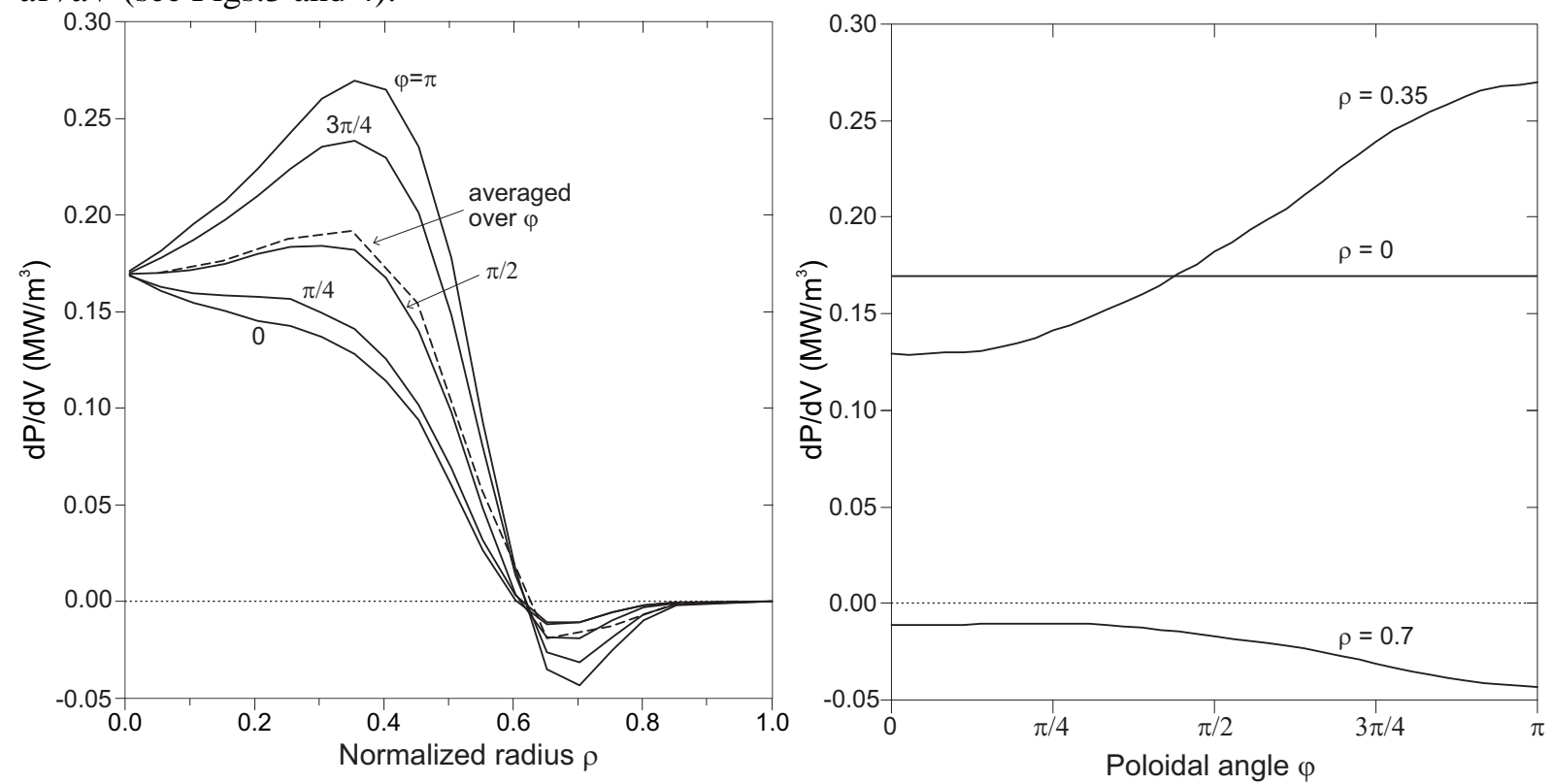

Figure 5. Left plot: radial profile of the net EC wave power density $d P / d V$ from RAYTEC for the reference toroidal geometry $\left(A_{\text {ref }}=3.1, \kappa_{\text {ref }}=1.7\right), R_{w}=0.8$, and the advanced $\mathrm{T}$ profile, for various poloidal angles $\varphi$, as well as the poloidal average of $d P / d V$ (dashed curve); right plot: the dependence of $d P / d V$ on the poloidal angle for three radial positions: in the plasma core $(\rho=0)$, at an intermediate radius $(\rho=0.35)$, and in the range of profile reversal $(\rho=0.7)$.

In the following, only the poloidally averaged $d P / d V$ profiles which are the ones of relevance for 1D transport studies, will be further discussed. Figs. 6(a) and 6(b) illustrate the results for $A_{\text {ref }}=3.1$ and different values of elongation $\left(\kappa=1,1.35,2.05\right.$ as well as $\left.\kappa_{\text {ref }}=1.7\right)$ for the advanced and the parabola-like temperature profiles. In Figs. 6, results from proto-RAYTEC, the preliminary version of the code written for cylindrical geometry and elliptical plasma cross-section, are also shown. For the limiting case of circular cross-section $(\kappa=1)$ the exact result as obtained from EXACTEC is also plotted, showing a perfect agreement with the one from RAYTEC. 

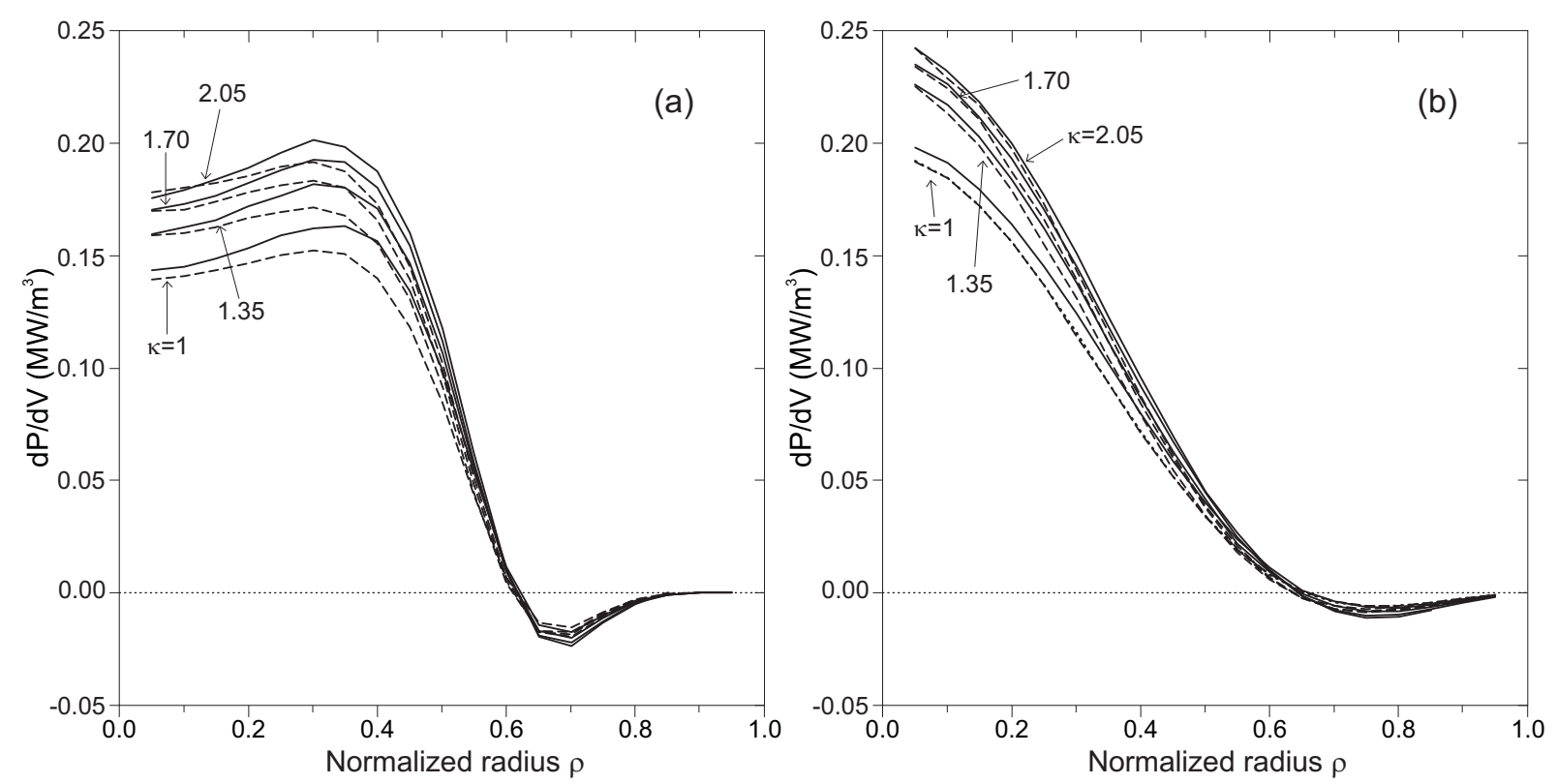

Figure 6. Net EC wave power density $d P / d V$ from RAYTEC for $A_{\text {ref }}=3.1$, reflection coefficient $R_{w}=0.8$, and "advanced" (left plot (a)) and parabola-like (right plot (b)) temperature profiles, for various values of plasma elongation; for comparison, the results for cylindrical geometry obtained using proto-RAYTEC (dashed), are also shown.

From Figs. 6 it is seen that in the core plasma $d P / d V$ increases with elongation by about $25 \%$ when going from the circular plasma to the highly elongated case $\kappa=2.05$. In the cooler outer layers elongation tends to enhance the profile reversal. This trend is mainly due to a growing homogeneisation of the specific intensity for increasing elongation of the plasma cross-section: whereas for a circular plasma, the EC wave power emitted by the hot inner plasma, upon reflection, is strongly concentrated onto the hot core and re-absorbed there, this symmetry effect weakens for increasing elongation and a larger part of the reflected wave power is absorbed in the intermediate and edge plasma. It is the assumption of specular reflection in RAYTEC that makes this effect particularly strong and also implies that RAYTEC generally yields a lower bound for $d P / d V$ with respect to realistic cases where wall reflection has a diffuse component (that also leads to a homogeneisation of the radiation); in fact, the assumption of specular reflection maximizes the effective optical thickness of the plasma for given geometry and plasma parameters [11]. This tendency, and hence the increase of $d P / d V$ with $\kappa$, gradually reduces also for decreasing specular wall reflection. However, a slight increase of $d P / d V$ related to the decrease of the effective optical thickness of the plasma with increasing elongation (see also Sect.3.1) is present in all cases.

A similar effect is observed when going from a cylindrical plasma to toroidal geometry. As seen in Fig.6, whereas the values of $d P / d V$ at the plasma centre are almost unchanged, toroidicity, and in particular the inhomogeneity of the magnetic field, enhances $d P / d V$ for intermediate radii as expected from the results of Sect.3.1.

The detailed dependence of $d P / d V$ on aspect ratio $A$ for a central $(\rho=0.05)$ and an intermediate $(\rho=0.4)$ plasma position is shown in Fig.7 for the two $T$-profiles and two plasma elongations, $\kappa=1$ and $\kappa_{\text {ref }}=1.7$. From this figure it is seen that (i) $d P / d V$ increases with the elongation for a given value of $A$, as already observed in Figs.6, and (ii) the toroidal effects, mainly the correction to the ray path length, effectively decrease the optical distance of the inner plasma from the edge with decreasing $A$ so that $d P / d V$ slightly increases. The inhomogeneity of the toroidal magnetic field enhances the geometrical effect for intermediate $(\rho \approx 0.4)$ plasma radii, increasing $d P / d V$, while it tends to reduce $d P / d V$ for plasma positions close to the magnetic axis due to a slightly higher overall re-absorption. As a general result, the variation of the net EC wave power loss with aspect ratio is small (less than $15 \%$ when comparing the results for large aspect ratio, i.e. the cylindrical limit, with those for low $A$ ). 

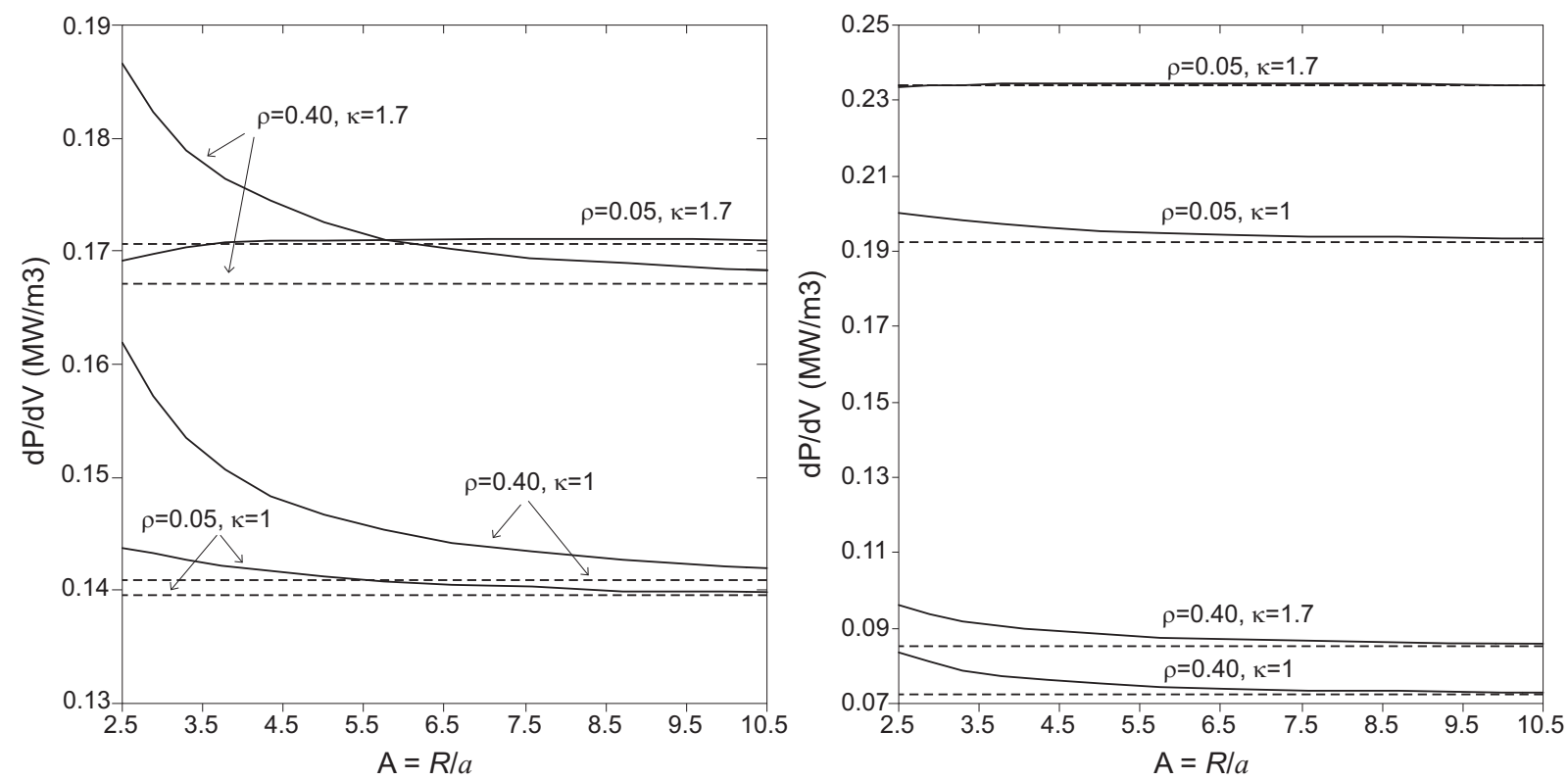

Figure 7. Net EC wave power density $d P / d V$ versus aspect ratio $A$ at $\rho=0.05$ and $\rho=0.40$, from RAYTEC for advanced (left plot) and parabola-like (right plot) temperature profiles: circular plasma cross-section $(\kappa=1)$ and reference elongation $\kappa_{\text {ref }}=1.7$; the wall reflection coefficient is $R_{w}=0.8$. The asymptotic values reached in the cylindrical limit are indicated by dashed lines.

In Figs.8a and $8 \mathrm{~b}$, a direct comparison of the radial profiles of $d P / d V$ as obtained using RAYTEC with those calculated with CYTRAN, EXACTEC and the locally applied Trubnikov formula (LATF) as given in the Appendix, is presented for the reference aspect ratio $A_{\text {ref }}=3.1$ and for a comparatively large value, $A=4.5$, of the aspect ratio, both associated with the reference elongation $\kappa_{\text {ref }}=1.7$ and with a circular cross section $(\kappa=1)$; results for both the advanced and the parabola-like temperature profile with a wall reflection coefficient $R_{w}=0.8$ are shown. From these one can infer that, overall, the simpler models produce quite good approximations to $d P / d V$ for toroidal geometries of practical interest. In the hot plasma core, with respect to RAYTEC, the analytical LATF (i) tends to underestimate $d P / d V$ for the parabola-type $T$-profile, this effect becoming somewhat larger for increasing elongation of the cross-section and larger aspect ratio, (ii) for the advanced $T$-profile, on the other hand, approximates quite closely the values of $d P / d V$ for a circular cross-section while for elongated cross-sections and in particular for large $A$, the results are again somewhat smaller than those of RAYTEC. Of course, the LATF profiles are throughout monotonic and LATF does not describe the power absorption present at the edge. Generally, in the outer part of the plasma LATF yields the largest values for $d P / d V$. EXACTEC, in all cases deviating from circularly cylindrical geometry, yields an underestimate of $d P / d V$ in the plasma core which is the larger the stronger the deviation is (see also Figs.6), due to the fact that it overestimates the overall optical thickness of the system (as EXACTEC does also with respect to models that rely on isotropisation of the radiation and hence rather are appropriate in the presence of a strong diffuse component in wall reflection (cf. [11]); for the reference case this underestimate, compared to RAYTEC, approaches $20 \%$. On the other hand, CYTRAN, assuming the radiation field to be isotropic, in fact, generally yields higher values of $d P / d V$ than RAYTEC, in the core plasma by typically $20 \%$, and generates a somewhat too peaked power density profile. 

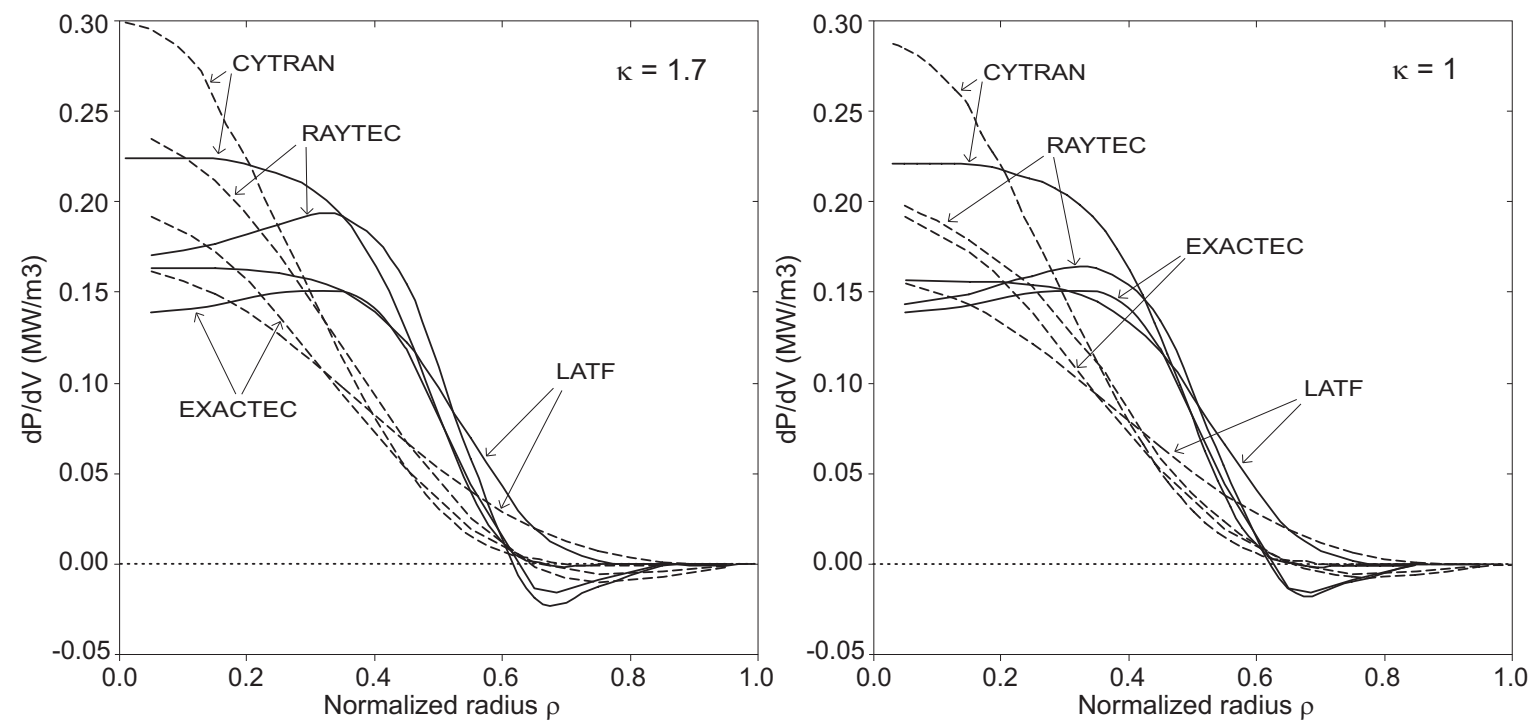

Figure 8a. Comparison of CYTRAN, EXACTEC and LATF results for $d P / d V$ with those obtained from RAYTEC for ITER-like parameters and the reference aspect ratio $A_{\text {ref }}=3.1$ : advanced temperature profile (solid curves) and parabola-like temperature profile (dashed curves); the left plot shows the results for the reference plasma elongation $\mathrm{k}_{\mathrm{ref}}=1.7$ while the right plot refers to a circular plasma $(\mathrm{k}=1)$.
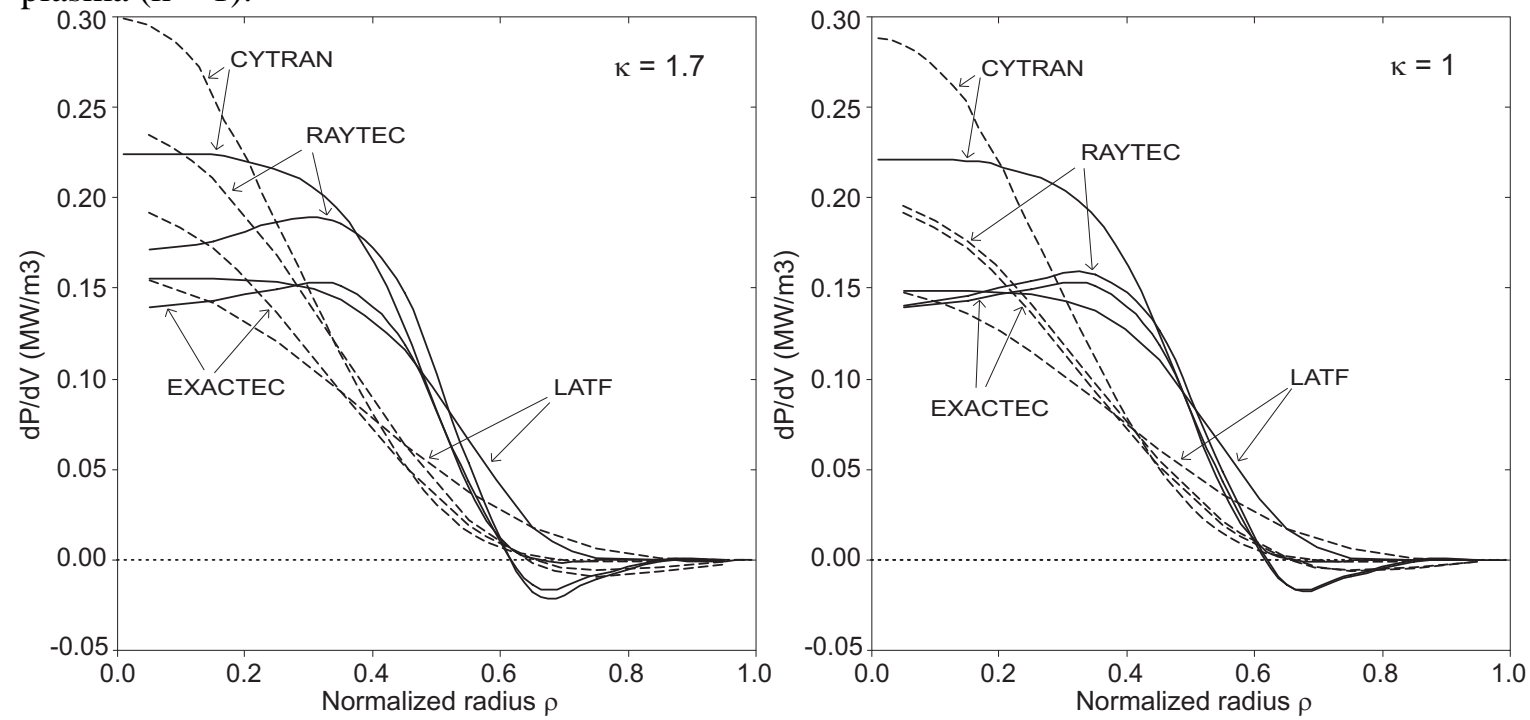

Figure 8b. Same as figure 8a, but for a large aspect ratio $(A=4.5)$

\subsection{Total EC power losses}

Integrating $d P / d V$ as calculated employing RAYTEC over the plasma volume, one readily obtains the total EC power loss $P_{E C}$ from the plasma which can also be used to benchmark simpler models, in particular in view of the dependence of $P_{E C} / R$ on plasma aspect ratio and elongation. In the following, we compare the RAYTEC results with $P_{E C}$ as resulting upon numerical volume integration of $d P / d V$ calculated with CYTRAN [4], EXACTEC [10] and LATF (see Appendix) as well as with the results obtained from the global expression given by Albajar et al [3] and that suggested by Fidone et al [14] specifically for generalized parabola-like profiles adopting magnetic surfaces with elliptical cross sections and a model Shafranov shift; in addition, $P_{E C}$ as given by the analytical expression (A.4) of the Appendix, obtained by an approximate integration of the LATF formula for $d P / d V$, is considered.

The results are summarized in Figs.9a and 9b which show, respectively, the dependence of $P_{E C} / R$ on aspect ratio for both the advanced and the parabola-like profile and two values of the elongation $\kappa$ (circular cross section, $\kappa=1$, and $\kappa_{\mathrm{ref}}=1,7$ ), and that on elongation for the reference aspect ratio $A_{\text {ref }}=3.1$. As compared to the RAYTEC values of $P_{E C} / R$ which represent a lower bound to situations 
in which wall reflection is (partially) diffusive, the deviations of the results generated by the other models do not exceed about $25 \%$. Also most of the parameter dependences are qualitatively similar. There are, however, striking differences between these models:

i) From Figs.9, it is transparent that the volume-integrated analytical LATF yields quite accurate results for the total EC power loss with a tendency to be on the high side for small $A$, that is strong toroidicity, then exceeding, for the parabola-like profile, even slightly the values given by CYTRAN (which relying on isotropisation of the radiation is expected to yield rather an upper bound for $P_{E C}$ ). Furthermore, LATF generates a slightly steeper decrease of $P_{E C} / R$ with increasing $A$ than most other models. While the values generated by the LATF based analytical expression (A.4), for $A_{\text {ref }}=3.1$, are generally just by about $2 \%$ higher than those obtained by numerical integration, this deviation increases somewhat with aspect ratio.

ii) CYTRAN results depend only very weakly on aspect ratio and moderately on elongation, the trend being right. One can conjecture that for small $A$ and/or large $\kappa$ it does actually not provide an absolute upper bound for $P_{E C}$.

iii) The EXACTEC model which is exact for circularly cylindrical geometry and specular wall reflection and does not include any geometry dependence, generally yields the lowest values of $P_{E C}$ and can be taken as providing an absolute lower bound for $P_{E C}$. Since the reduction of the effective optical thickness of the plasma for increasing toroidicity and elongation is not covered by EXACTEC, it increasingly underestimates $P_{E C}$ in this case. For the reference geometry this underestimate may amount to about $20 \%$.

iv) The global formula due to Albajar et al, as a general tendency, produces somewhat low results for $P_{E C}$, in particular for the advanced profile in the cylindrical limit where the value given is even lower than the one produced by EXACTEC. The parameter dependence on aspect ratio and elongation show the right trend.

v) The results from the formula suggested by Fidone et al, applicable only to the case of the parabola-like plasma profile, show a considerably stronger dependence on aspect ratio than all other models, and for the dependence on elongation a decrease of $P_{E C}$ with increasing $\mathrm{\kappa}$ is found which is inconsistent with the actual impact of elongation.

vi) For RAYTEC results one observes the strongest increase of $P_{E C}$ on elongation, especially for values of $\kappa$ just above 1 , which again can be traced back to the fact that for specular wall reflection there is a sizeable homogeneisation of the radiation when circular symmetry is disturbed.
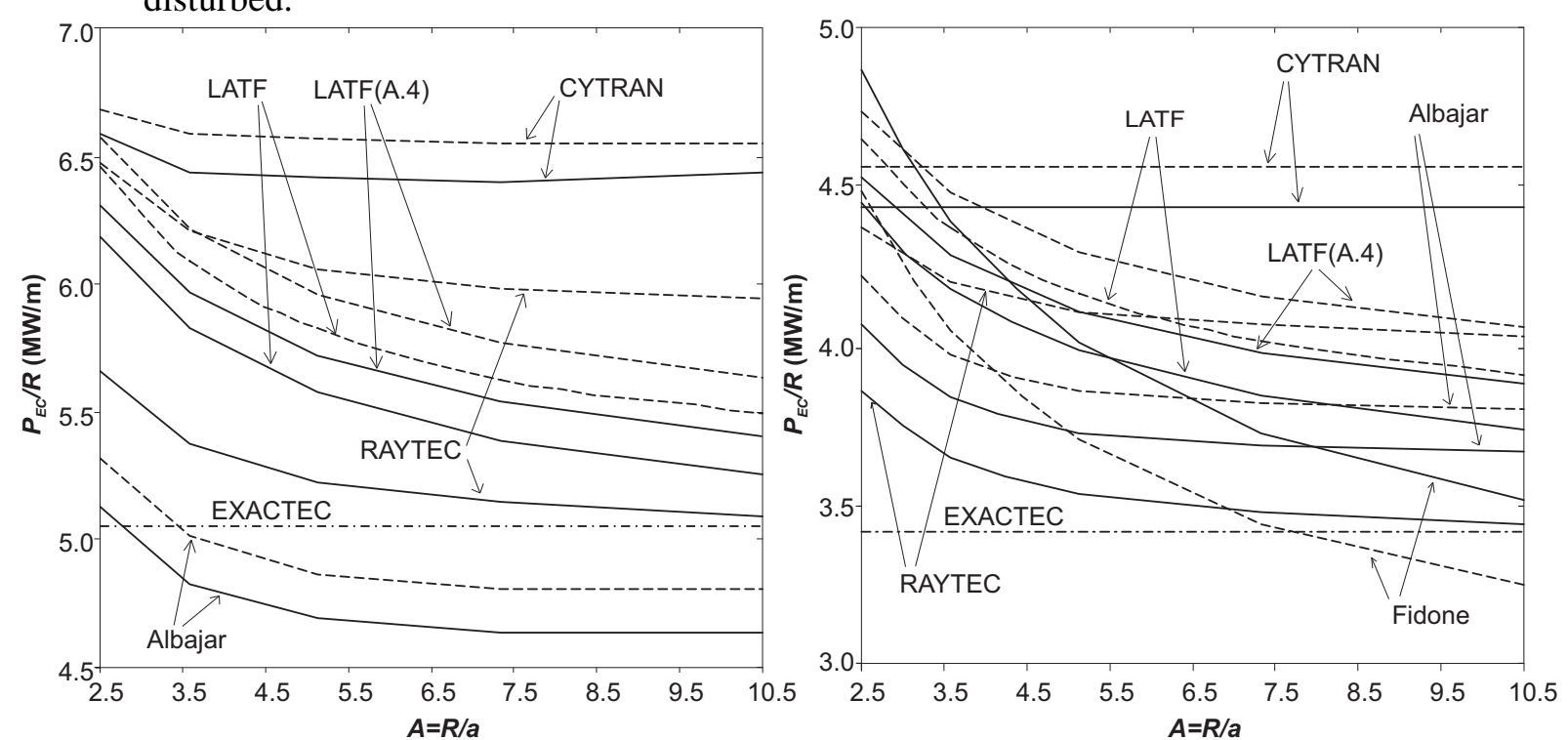

Figure 9a. Comparison of the total EC wave power emitted per toroidal length $P_{E C} / R$ versus aspect ratio $A$ as obtained by integrating $d P / d V$ calculated using CYTRAN, EXACTEC and LATF numerically over the plasma volume, as well as that derived from the global formulae given by Albajar et al and by Fidone et al and from the approximate analytical expression (A.4) based on 
LATF, with the volume-integrated RAYTEC results for ITER-like parameters and circular cross section (solid curves) as well as the reference plasma elongation $\kappa_{\text {ref }}=1.7$ (dashed curves); left plot: advanced profile, right plot: parabola-like profile; the reflection coefficient is $R_{w}=0.8$.
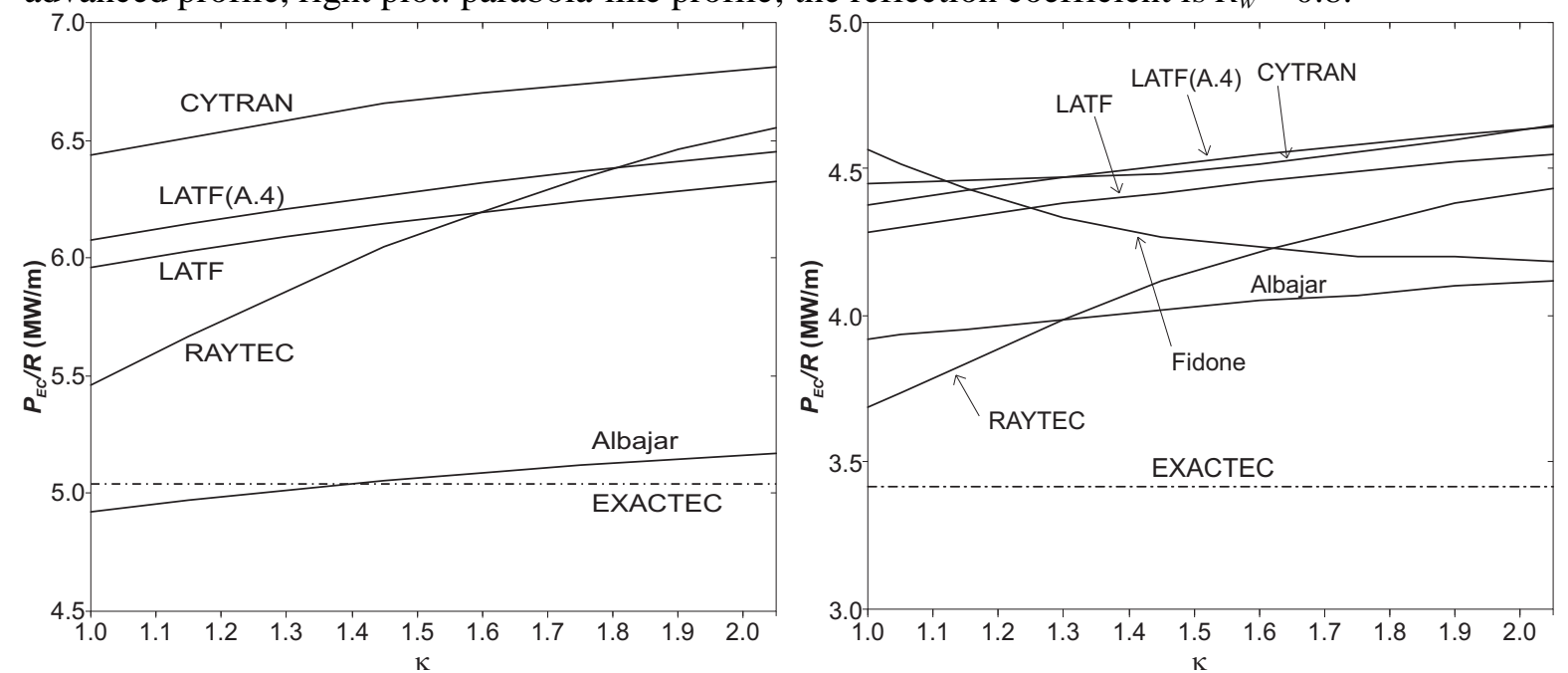

Figure 9b. Same as figure 9a, but for the dependence on elongation $\kappa$ at fixed $A_{\text {ref }}=3.1$

\section{Conclusions}

Electron cyclotron (EC) wave losses have been recognized to be important in the local electron power balance of reactor-grade tokamak plasmas with core temperatures of $35 \mathrm{keV}$ or higher as anticipated for steady-state operation in ITER and DEMO and, therefore, can influence the temperature profile of these plasmas. This has motivated an effort to improve the modelling capability of the radial profile of EC power losses. As part of this effort, recently the code RAYTEC has been set up for general toroidal geometry assuming wall reflection to be specular. Systematic studies of the impact of plasma geometry on the angular dependence of the EC radiation field, on the profile of the net EC wave power density lost from the plasma and on the total EC power loss were performed for toroidal plasmas in ITER-like steady-state operation conditions. It was found that toroidicity and plasma elongation by themselves do not lead to an appreciable isotropisation of the radiation field. In the hot core, they enhance the net losses due to EC waves typically by $25 \%$ with respect to the equivalent circularly cylindrical plasma. This is not far from what is found to occur just through isotropisation of the wave field, e.g., by a diffuse component in wall reflection [11]. It is concluded that RAYTEC can be used with good accuracy (certainly considerably better than $25 \%$ locally) in 1D plasma transport calculations for ITER and DEMO. A comparison with the more simplified models underlying the computationally faster codes CYTRAN and EXACTEC as well as with an analytic formula ("LATF") constructed on the basis of Trubnikov's expression for the global EC power emission [6] has been made and has shown that the latter models do not deviate by more than about $20 \%$ and, hence, are still quite adequate for this aim although they do not describe geometry and profile effects as accurately. The analysis of the total EC power loss obtained by volume integration of the net local losses shows similar enhancements with increasing toroidicity and plasma elongation for RAYTEC; the results found using CYTRAN, EXACTEC and LATF as well as explicit expressions due to Albajar et al [3] and Fidone et al [14], and one proposed on the basis of the LATF approach (which separates profile effects from those of geometry and the global plasma parameters) are within $25 \%$ of the RAYTEC values, but again do reflect the actual impact of geometry only in part.

\section{Appendix: The Locally Applied Trubnikov Formula (LATF)}

For a simplified evaluation of the total EC radiation loss from fusion plasmas, global models pertinent to homogeneous slabs and/or cylinders and possibly accounting for corrections to also cover wall reflection, a finite aspect ratio and elongation of the plasma cross section can be used in an ad hoc way. Tentatively, even profile effects of the radiation loss can be addressed by locally applying a global model, that is, by calculating the radiation loss per unit volume as following from the global 
model pointwise in the plasma using the local values of both density and temperature $[14,15]$. The advantage of this obviously heuristic approach is that one obtains an explicit analytical formula for the net EC wave power density $d P / d V$ in terms of local and global plasma parameters.

Here we consider specifically Trubnikov's well-known global formula for the EC radiation loss from a homogeneous plasma in circularly cylindrical geometry, adjusted to include an estimate of toroidal effects, $[2,6]$,

$$
P_{E C}^{(T r u b)}=C^{(T r u b)}\left(R_{w}\right) R a^{3 / 2} n^{1 / 2}(T B)^{5 / 2} M(T) G(A, T)
$$

where

$$
C^{(T r u b)} \equiv 8.2 \times 10^{-6}\left(1-R_{w}\right)^{1 / 2}
$$

with again $R_{w}(\leq 1)$ the wall reflection coefficient and $A \equiv R / a$ the plasma aspect ratio; furthermore, the multiplicator

$$
M(T) \equiv \frac{K_{3}(\mu)}{K_{2}(\mu)}=\frac{1+1.93030 / \mu}{1-0.58167 / \mu} \quad \text { with } \quad \mu \equiv \frac{m c^{2}}{T} \gg 1
$$

( $K_{v}(\mu)$ denoting the Macdonald function, $m$ the electron mass and $c$ the velocity of light accounts for relativistic effects, and the heuristic correction factor

$$
G(A, T)=\left(1+\frac{18}{A \sqrt{T}}\right)^{1 / 2}
$$

approximately quantifies the enhancement of the radiated EC power due to the reduction of the effective size of the plasma caused by the topological properties of and the inhomogeneity of the magnetic field in toroidal geometry. The units in the dimensional formula (A.1a) are MW, $\mathrm{m}, 10^{20}$ $\mathrm{m}^{-3}, \mathrm{keV}$, and Tesla.

On dividing $P_{E C}{ }^{(T r u b)}$ by the plasma volume $V=2 \pi^{2} R a^{2}$ and taking both density and temperature as functions of the radial coordinate $r$, one can transform Eq.(A.1) into an explicit expression for the locally radiated EC power per unit volume, $d P / d V$, for a toroidal plasma with circular cross section, viz.,

$$
\frac{d P}{d V}=\frac{C^{(T r u b)}}{(2 \pi)^{2}} a^{-1 / 2}(n(r))^{1 / 2}(T(r) B)^{5 / 2} M(T) G(A, T) \quad \mathrm{MW} / \mathrm{m}^{3}
$$

To construct a model that covers approximately also (vertically) elongated cross sections, one can use the fact that for a plasma slab, formula (A.2a) is likewise valid if $a$ is replaced by the thickness of the slab $[2,6]$. Since for large elongation $\kappa$ twice the minor plasma radius $a$ tends to correspond to the thickness of a slab, a somewhat arbitrary but reasonable way to achieve this is to make in Eq.(A.2a) the replacement

$$
a \rightarrow a \kappa^{1 / 3}
$$

In fact, the substitution (A.2b) implies that $a$ is replaced by $2 a$ for $\kappa=8$. With the scaling of Eq.(8a), one then has $d P / d V \sim \kappa^{1 / 12}$. As far as the dependence on the aspect ratio $A$ is concerned, it is reasonable to identify $A$ in Eq.(A.1d) with the aspect ratio of the hot core plasma from which most of the radiation is emitted, that is to make the replacement

$$
A \rightarrow 2 A
$$

to maintain the meaning of $A$ as the aspect ratio of the plasma as a whole. This effectively implies to replace in Eq.(A.1d) the factor 18 by 9. Formula (A.2a) modified according to the relations (A.2b) and (A.2.c) is referred to as the locally applied Trubnikov formula (LATF). It provides an explicit expression for $d P / d V$ and is in accordance with the fact that the radial profile of the EC wave power density radiated is most strongly affected by the radial profile of the electron temperature, the effect of the density profile being comparatively weak.

However, the LATF approach, for monotonic plasma profiles, necessarily generates a monotonic profile also for $d P / d V$; this is actually not always appropriate as exemplified by the advanced plasma profile considered in Sect.3. Since the product $M(T) G(A, T)$ in Eq.(A.2a) describing relativistic and aspect ratio effects is only weakly dependent on temperature and, hence, the temperature profile, the 
profile of $d P / d V$ is close to invariant with respect to changes of the global temperature and density at fixed profile parameters. Note furthermore that $d P / d V$ when taken according to Eqs.(A.2) is unconditionally positive and consequently the EC wave power absorption occurring at the plasma edge for sufficiently high wall reflectivity (see, eg., [12]) is not accounted for.

Integrating Eq.(A.2) over the plasma volume again yields an evaluation of the total EC radiated power with the profile effects of both density and temperature accounted for according to the LATF approximation. If one approximates the cross section of the magnetic surfaces by concentric ellipses (as also done in the RAYTEC applications presented in this paper) and disregards the comparatively weak spatial dependence of the product of the relativistic multiplicator $M$ and the toroidal correction factor $G$, this integration can also be carried out analytically for a quite general class of radial plasma profiles (although more limited than that originally considered in Sect.3), that is for

$$
n(\rho)=n(0)\left(1-\rho^{\beta}\right)^{\gamma_{n}} \text { and } T(\rho)=T(0)\left(1-\rho^{\beta}\right)^{\gamma_{T}}
$$

where $n(0)$ and $T(0)$ are the peak density and temperature, respectively, and $\beta, \gamma_{\mathrm{n}}$ and $\gamma_{\mathrm{T}}$ are positive indices that allow to cover a wide range of profile shapes. For the volume averages of the profiles $n(\rho)$ and $T(\rho)$ one readily obtains

$$
\langle n\rangle=\frac{\Gamma(1+2 / \beta) \Gamma\left(1+\gamma_{n}\right)}{\Gamma\left(1+2 / \beta+\gamma_{n}\right)} n(0) \quad \text { and } \quad\langle T\rangle=\frac{\Gamma(1+2 / \beta) \Gamma\left(1+\gamma_{T}\right)}{\Gamma\left(1+2 / \beta+\gamma_{T}\right)} T(0)
$$

where $\Gamma(x)$ is the $\Gamma$-function. If the volume integration of Eq.(A.2a) is performed disregarding the spatial dependence of the product $M(T) G(A, T)$, one obtains for the total EC radiated power

$$
P_{E C}\left(\beta, \gamma_{n}, \gamma_{T}\right)=\kappa^{5 / 6} \frac{\Gamma(1+2 / \beta) \Gamma\left(1+5 \gamma_{T} / 2+\gamma_{n} / 2\right)}{\Gamma\left(1+2 / \beta+5 \gamma_{T} / 2+\gamma_{n} / 2\right)} P_{E C}^{(T r u b)}(n, T \rightarrow n(0), T(0))
$$

upon taking account of the replacement (A.2b). The use of $T=T(0)$ in $M(T)$ and $G(A, T)$, in Eq.(A.4), is an approximation convenient in this context. Note that the additional factor $\kappa$ appearing in Eq.(A.4) is exact for the geometry of the magnetic surfaces under consideration since $\kappa$ here just enters as a constant scaling factor in the vertical direction.

As an alternative to Eq.(A.4), using Eqs.(A3b), one can also express $P_{E C}$ in terms of $\langle n\rangle$ and $\langle T\rangle$, if one rather uses $T=\langle T\rangle$ in $M(T)$ and $G(A, T)$. In this form profile effects are quantifiable in a way that is separated from variations of the overall plasma density and temperature as well as from those of aspect ratio $A$ and elongation $\kappa$, that is, of the plasma geometry.

Choosing $\beta=2$, Eq.(A.4) yields $P_{E C}$ for generalized parabola-like profiles,

$$
P_{E C}\left(\gamma_{n}, \gamma_{T}\right)=\left\{\begin{array}{c}
\frac{\kappa^{5 / 6}}{1+\left(\gamma_{n}+5 \gamma_{T}\right) / 2} P_{E C}^{(T r u b)}(n, T \rightarrow n(0), T(0)) \\
\kappa^{5 / 6} f\left(\gamma_{n}, \gamma_{T}\right) P_{E C}^{(T r u b)}(n, T \rightarrow\langle n\rangle,\langle T\rangle)
\end{array}\right.
$$

with

$$
f\left(\gamma_{n}, \gamma_{T}\right) \equiv \frac{\left(1+\gamma_{n}\right)^{1 / 2}\left(1+\gamma_{T}\right)^{5 / 2}}{1+\left(\gamma_{n}+5 \gamma_{T}\right) / 2}
$$

where, to obtain Eq.(A.5c), Eqs.(A.3b) have also been used.

For flat density profiles $\left(\gamma_{n}=0\right)$, Eq.(A.4) reduces to

$$
P_{E C}\left(\gamma_{n}, \gamma_{T}\right)=\left\{\begin{array}{c}
\kappa^{5 / 6} \frac{\Gamma(1+2 / \beta) \Gamma\left(1+5 \gamma_{T} / 2\right)}{\Gamma\left(1+2 / \beta+5 \gamma_{T} / 2\right)} P_{E C}^{(T r u b)}(n, T \rightarrow n(0), T(0)) \\
\kappa^{5 / 6} g\left(\beta, \gamma_{T}\right) P_{E C}^{(T r u b)}(n, T \rightarrow\langle n\rangle,\langle T\rangle)
\end{array}\right.
$$

with

$$
g\left(\beta, \gamma_{T}\right) \equiv \frac{\Gamma\left(1+5 \gamma_{T} / 2\right)}{[\Gamma(1+2 / \beta)]^{3 / 2} \Gamma\left(1+2 / \beta+5 \gamma_{T} / 2\right)}\left[\frac{\Gamma\left(1+2 / \beta+\gamma_{T}\right)}{\Gamma\left(1+\gamma_{T}\right)}\right]^{5 / 2}
$$


Let us, for example, compare the total EC wave power loss $P_{E C}$ as resulting for the parabola-like profile considered in Sect.3 (i.e., $\beta=2 ; \gamma_{n}=0.1$ and $\gamma_{T}=1.5$ ) with the value obtained for the case of homogeneous electron temperature, $\gamma_{T}=0$ : Eq.(A.5c) readily yields for this case a profile enhancement factor of the power loss $\left[f\left(\gamma_{n}=0.1, \gamma_{T}=1.5\right) / f\left(\gamma_{n}=0.1, \gamma_{T}=0\right)\right] \approx 2.16$. This value is, respectively, $11 \%$ and $17 \%$ lower than the actual enhancements as resulting from [10], Tables 1 and 2 , for $\langle T\rangle=9 \mathrm{keV}$ and $\langle T\rangle=18 \mathrm{keV}$, obtained for circularly cylindrical geometry applying the EXACTEC code, showing a reasonable accuracy of the approach for this case. Starting from Eq.(A.6c) one can evaluate approximate profile enhancement factors both for the parabola-like and the advanced profile (characterised by $\beta=5.4$ and $\gamma_{T}=8$ ) of Sect.3. For the enhancements factors with respect to a homogeneous plasma one has, respectively, $g\left(\beta=2, \gamma_{T}=1.5\right)=2.08$ and $g\left(\beta=5.4, \gamma_{T}=8\right)=2.86$. Neglecting the weak density inhomogeneity, hence, lowers the enhancement factor for the parabola-like profile just by $4 \%$. The enhancement of $P_{E C}$ for the advanced profile is by a factor 1.38 larger than that for the parabola-like one (which is close to the value apparent from Figs.9 of Sect.3.c). The enhancement factor obtained for the advanced profile is, respectively, by $18 \%$ and $25 \%$ lower than the actual values reported in [10], Tables 1 and 2 , for $\langle T\rangle=9 \mathrm{keV}$ and $\langle T\rangle=18$ $\mathrm{keV}$; the relative enhancement with respect to the parabola-like profile results to be, respectively, by $4 \%$ and $6 \%$ too low.

\section{References}

[1] Albajar F et al 2005 Importance of Electron Energy Transport in ITER Nucl. Fusion 45642

[2] Bornatici M, Cano R, De Barbieri O and Engelmann F 1983 Electron Cyclotron Emission and Absorption in Fusion Plasmas Nucl. Fusion 231153

[3] Albajar F, Johner J and Granata G 2001 Improved Calculation of Synchrotron Radiation Losses in Realistic Tokamak Plasmas Nucl. Fusion 41665

[4] Tamor S 1981 A Simple Fast Routine for Computation of Energy Transport by Synchrotron Radiation in Tokamaks and Similar Geometries Report SAI-023-81-189-LJ/LAPS-72 (La Jolla, CA: Science Applications) pp 1-26

[5] Kukushkin A B 1992 Heat Transport by Cyclotron Waves in Plasmas with Strong Magnetic Field and Highly Reflecting Walls in Plasma Physics and Controlled Nuclear Fusion Research 1992 (Proc. 14th Int. Conf. Wuerzburg, 1992), Vol 2, p. 35, IAEA, Vienna (1993)

[6] Trubnikov B A 1979 Universal Coefficients for Synchrotron Emission from Plasma Configurations Rev. Plasma Phys. vol 7 ed M A Leontovich (New York: Consultants Bureau) pp 345

[7] Robinson P A 1985 Synchrotron Emission and Absorption by Relativistic Loss Cone and AntiLoss Cone Distributions Plasma Phys. Control. Fusion 271037

[8] Albajar F, Bertelli N, Bornatici M and Engelmann F 2007 Electron Cyclotron Absorption in High-Temperature Plasmas: Quasi-Exact Analytical Evaluation and Comparative Numerical Analysis Plasma Phys. Control. Fusion 4915.

[9] Albajar F, Bornatici M and Engelmann F 2007 Electron Cyclotron Wave Power Loss in Fusion Plasmas: a Model Comparison Nucl. Fusion 471101

[10] Albajar F, Bornatici M and Engelmann F 2002 Electron Cyclotron Radiative Transfer in Fusion Plasmas Nucl. Fusion $\mathbf{4 2} 670$

[11] Albajar F, Bornatici M, Engelmann F and Kukushkin A B 2009 Benchmarking of Codes for Calculating Local Net EC Power Losses in Fusion Plasmas Fusion Science and Techn. 5576

[12] Tamor S 1983 Calculation of Energy Transport by Cyclotron Radiation in Fusion Plasmas Nucl. Technol. Fusion 3293

[13] Albajar F, Bornatici M and Engelmann F 2008 Recent Progress in Electron Cyclotron Radiative Transport Modelling of Fusion Plasmas in View of ITER and DEMO Applications Proc. $15^{\text {th }}$

Joint Workshop on ECE and ECRH (Yosemite National Park, CA) World Scientific Publishing, Singapore (2009)

[14] Fidone I, Meyer R L, Giruzzi F and Granata G 1992 Temperature Dependence of Synchrotron Radiation Loss in Inhomogeneous Tokamak Plasmas Phys. Fluids B 44051 
RAYTEC: a new code for EC radiative transport modeling of fusion plasmas

[15] Tamor S 1988 Synchrotron Radiation Loss from Hot Plasma Nucl. Instr. Methods Phys. Res. A271 37 